\title{
Analysis of Resource Sharing in Transparent Networks
}

\author{
Dimitri Staessens · Didier Colle • Mario Pickavet • Piet Demeester
}

Received: date / Accepted: date

\begin{abstract}
Transparent optical networking promises a cost-efficient solution for future core and metro networks because of the efficacy of switching high-granularity trunk traffic without opto-electronic conversion. Network availability is an important performance parameter for network operators, who are incorporating protection and restoration mechanisms in the network to achieve competitive advantages. This paper focuses on the reduction in Capital Expenditures (CapEx) expected from implementing sharing of backup resources in pathprotected transparent networks. We dimension a nationwide network topology for different protection mechanisms using transparent and opaque architectures. We investigate the CapEx reductions obtained through protection sharing on a population of 1000 randomly generated biconnected planar topologies with 14 nodes. We show that the gain for transparent networks is heavily dependent on the offered load, with almost no relative gain for low load (no required parallel line systems). We also show that for opaque networks the CapEx reduction through protection sharing is independent of the traffic load and shows only a small dependency on the number of links in the network. The node CapEx reduction for high load (relative to the number of channels in a line system) is comparable to the CapEx reduction in opaque OTN systems. This is rather surprising as in OTN systems the number of transceivers and linecards and the size of the OTN switching matrix all decrease, while in transparent networks only the degree of the ROADM (number and size of WSSs in the node) de-
\end{abstract}

Ghent University - iMinds

Department of Information Technology

Gaston Crommenlaan 8 bus 201, 9050 Ghent Belgium

E-mail: firstname.lastname@intec.ugent.be creases while the number of transponders remains the same.

Keywords Network design - Transparent network · Resource sharing · Resiliency

\section{Introduction}

Recent advances made the availability of ultra long haul Wavelength Division Multiplexing (WDM) transmission systems possible at extremely competitive prices. This has opened up new perspectives in the design of cost-effective optical transport networks. According to the utilization of OEO devices, three types of networks are identified: opaque, transparent, and translucent networks[1]. An opaque network is characterized by OEO regenerations at every node. In a transparent network the signal bypasses the OEO devices during its transmission. Translucent networks are situated somewhere inbetween, where some paths require intermediate OEO regeneration. Introduction of transparency in the network allows for a reduction in expensive opticalto-electrical-to-optical (OEO) conversion and effectively reduces the total network cost [2].

One of the key issues in transparent networks is due to the increased length the signal travels without electronic regeneration. Each amplifier adds noise to the line, meaning that signals will have to be regenerated at some point. In addition to this, longer lightpaths are sensitive to various nonlinear optical impairments, especially when considering high data rates $(>10 \mathrm{~Gb} / \mathrm{s})$. The maximum transparent length (MTL) of a system puts a limit on the size of a completely transparent network. One way of dealing with the impairments in 
transparent networks is to introduce Islands of Transparency [3]. This is a part of the network where all possible transparent lightpaths are feasible end-to-end. Connections leaving a transparent island are regenerated at the edge. More recent work, however, has turned towards optimization of the entire network, taking into account the network node architectures [4].

This paper presents results for the cost estimation (Capital Expenditures or CapEx) for opaque and transparent networks, dimensioned for a given traffic demand and using different protection schemes. Dimensioning the network requires us to calculate routes and allocate the wavelengths to be used for each traffic demand, called Routing and Wavelength Assignment (RWA)[5]. Optimized RWA for minimizing resource usage and blocking in wavelength-switched networks is NP-complete[6]. For the unprotected and dedicated protection solutions, we follow an R+WA scheme: we first calculate the route and then assign an available wavelength for that route using first fit wavelength assignment. Due to the size of our dataset, requiring thousands of dimensionings, advanced algorithms (for instance based on k-shortest paths [7]) are unfeasible. Therefore, the paths are calculated using Dijkstra's algorithm in the unprotected case, the algorithm by Suurballe and Tarjan [8] is used for link-disjoint and node-disjoint cases. For mesh shared protection, optimized RWA becomes a very complex optimization problem which is well researched. ILP formulations [9] and approximation algorithms [10] have been proposed.

In previous work [11] we have shown that sharing backup resources has far less CapEx benefits in transparent networks compared to traditional opaque networks. In this work, we improve and extend that study considerably through simulation on random generated topologies and incorporation of the wavelength continuity constraint.

\section{Node Architectures and Cost Model}

The considered transparent node implementation is based on the well-known broadcast-and-select ROADM architecture. Fig. 1 shows a 3-degree ROADM, meaning it has 3 input/output fibers (typically labelled North, South, East, also see simplified structure in the bottom inset) and 3 add/drop terminals, one for each direction. From the add/drop terminal, each transponder is connected via a wavelength multiplexer/de-multiplexer (e.g. Arrayed Waveguide Grating, AWG) to a fixed output direction of the ROADM. This is called directionality and such an architecture is called directional. If a particular wavelength is not equipped in the terminal attached to a specific output fiber, it cannot be used

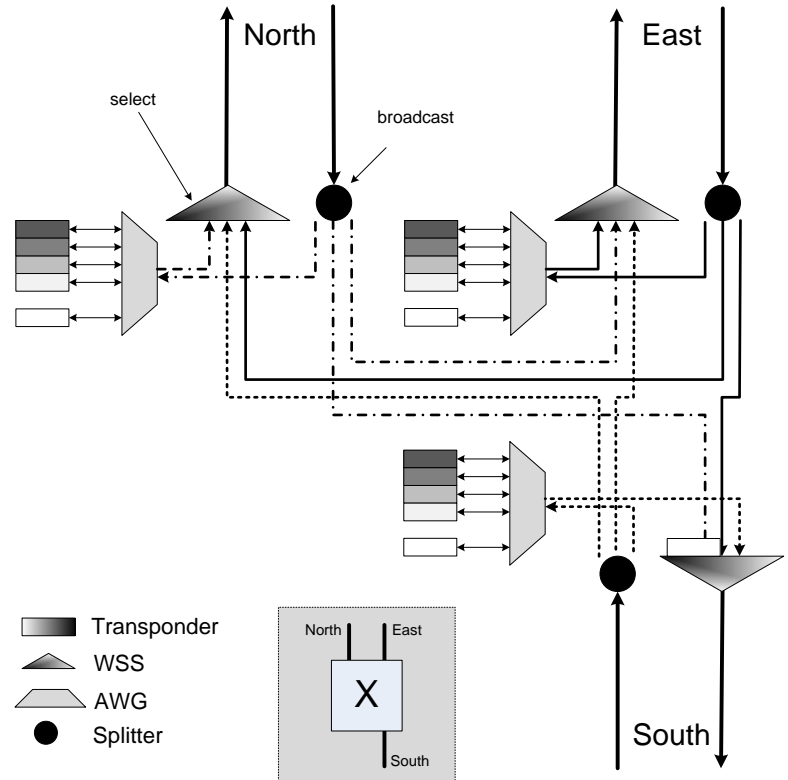

Fig. 1 Reconfigurable Optical Add-Drop Multiplexer Architecture

for add/drop at that particular fiber. The advantage is that there is no need for switching equipment in the add/drop terminals which reduces the node cost significantly. If we follow the lightpath on an incoming fiber for an $n$-degree ROADM, it is split to $n$ directions: the $n-1$ other output fibers and the drop terminal. In our example, traffic coming from the Northern input is split to the Northern add/drop terminal and to the Southern and Eastern output fibers. In the drop terminal it is demultiplexed to the transponders. If we follow the lightpath in the other direction, starting from the transponder output, it is first combined with the output from other transponders through a multiplexer (AWG) and then selected by a Wavelength Selective Switch (WSS) towards the output fiber. WSSs are bidirectional devices which have 1 input/output port and a number of output/input ports from which they can demultiplex or multiplex multiple wavelengths while selecting from each input port. The WSS is used to relieve wavelength contention, i.e. if multiple input ports forward traffic on the same wavelength, select the correct one. It could be replaced by a wavelength blocker/filter, which may further reduce costs, however, it seems that commercially it makes little sense as most ROADMs on the market are based on WSSs. Commercially available WSSs are limited in the number of ports, usually to $10(1 \mathrm{x} 9)$. A commercial 20 port WSS was launched by Finisar in 2011, a commercial 24 port WSS was introduced by Oclaro at OFC 2012 and the port count of experimental devices increases rapidly, with 1x43 already demonstrated as early as 2009 [12]. ROADMs 


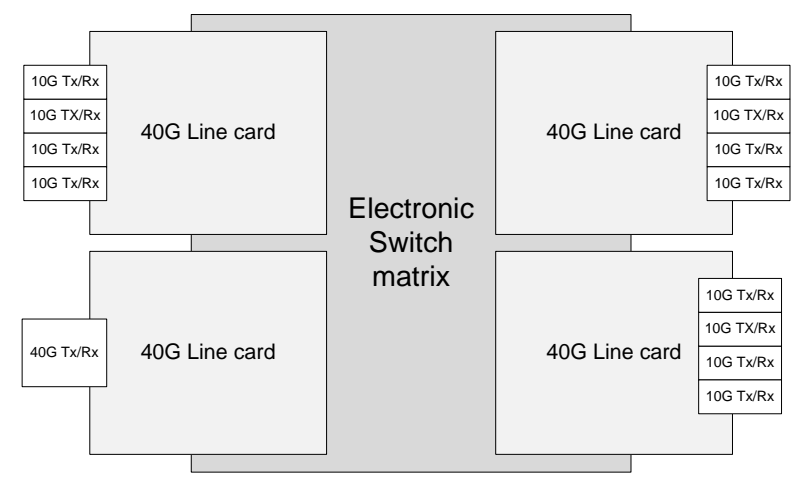

Fig. 2 Electronic cross-connect

which have degree $N \leq 8$ use 1x9 WSSs and ROADMs with degree $9 \leq N \leq 19$ use $1 \times 20$ WSSs.

The considered opaque solution is an electronic crossconnect and consists of three major functional components: The switch matrix (or basic node), the line cards and the transceivers (Figure 2). The switch matrix performs all switching functions and has a certain number of available line card slots. The line cards perform a conversion function from the transceivers to the switch fabric. This allows the switch fabric to operate independently of the protocol and support for instance 10GE (Ethernet), OTU2 (OTN) and STM-64 line cards. It is also possible for line cards to support multiple transceivers at lower data rates (for instance 4x10G transceivers in a 40G line card). We consider the costs for an Optical Transport Network (OTN) node, including the basic node, interface cards and transceivers. For the transparent solution, the cost of the terminals, ROADM node, input amplifiers and transponders are included, as are the tributary interfaces. The cost of the transmission links is not considered in this study, because they will be the same in both solutions. The used cost model is based on the models from [13] [14] [15] and is being updated in the STRONGEST [16] project. The cost values used in this text are given in Table 1.

\section{Considered Recovery Methods}

Because resource sharing optimization is a complex problem that demands considerable computation resources to compute to optimality, we use the dimensioning for restoration as a compromise for an optimized shared mesh protection scheme. The drawback of this approach is that it is not feasible to implement restoration on the transparent architecture due to directionality: in the architecture as shown in Figure 1 we cannot reuse a transponder if its outgoing link fails, because it is dedi-
Table 1 Cost model

\begin{tabular}{ll}
\hline Equipment & Cost \\
\hline \hline WDM layer & \\
\hline Transponder 10G grey & 0.1 \\
Transponder 10G 2000km & 1.2 \\
$N$ degree ROADM $(N \leq 8)$ & $N * 9.2$ \\
$N$ degree ROADM $(9 \leq N \leq 19)$ & $N * 11.8$ \\
\hline OTN Layer & \\
\hline Transceiver grey 10G & 0.1 \\
Transceiver 10G 2000km & 1.1 \\
linecard 10x10G & 16 \\
node 8 slot & 7 \\
node 16 slot & 14.3 \\
node 32 slot & 28.6 \\
node 64 slot & 67 \\
node 128 slot & 154 \\
\hline
\end{tabular}

cated to this one direction. This means we will have an underestimate of the true transponder cost of shared mesh protection and 1:1 protection in the transparent solution. In the approach we implemented, due to the possibility that the restoration path for a failed working path can use different outgoing links (as opposed to a single fixed one for 1:1 protection) we underestimate the transponder cost for shared mesh restoration more than we underestimate the transponder cost for 1:1 protection. This means that, when comparing 1:1 protection to shared mesh protection (approximated by restoration) in the transparent case, it is important to note that we have an overestimation of the benefits of protection sharing in transparent networks. Also, note that the $1+1$ protection scheme can be implemented on the ROADM architecture because all transponders are duplicated.

In summary, we consider the following protection schemes:

- Unprotected. All traffic is routed over the physical shortest paths, calculated using Dijkstra's algorithm, using 10G wavelengths.

- Link / node restored. This serves as a benchmark dimensioning for a shared mesh protection scheme. All traffic is routed over physical shortest paths. For each failure scenario (all possible single link failures for link restored and all possible link and node failures for node-restored) we calculate the required network resources required and determine the minimum which are needed to cover all of the failure scenarios[17].

- Link / node 1:1 protected. All traffic is routed over physical shortest cycles, calculated using the SuurballeTarjan algorithm [8] for link-disjoint. For node-disjoint, we run the same algorithm on a modified directed graph where each node is split in two nodes, one containing the incoming edges, one containing the out- 
going edges and a single directed edge is added between them from the node with the incoming edges to the node with the outgoing edges. The working path is the physically shorter half of the cycle.

- Link / node 1+1 protected. Uses the same paths as the 1:1 protected, only the traffic is duplicated and sent over both working and backup paths, meaning we also protect the transponders.

In all these scenario's, we use a two-step R+WA approach: we first determine the path using the algorithm detailes above, and then assign the appropriate wavelength(s) using first fit.

We evaluate the cost of these different protection schemes on a national backbone reference network (Fig. 3) with 14 nodes and 23 links. Each link has 80 wavelength channels available. The most relevant characteristics are given in Table 2 and the traffic matrix is given in 3 .

\begin{tabular}{ll}
\hline Node ID & Name \\
\hline 1 & Berlin \\
2 & Bremen \\
3 & Dortmund \\
4 & Düsseldorf \\
5 & Essen \\
6 & Frankfurt/Main \\
7 & Hamburg \\
8 & Hannover \\
9 & Köln \\
10 & Leipzig \\
11 & München \\
12 & Nürnberg \\
13 & Stuttgart \\
14 & Ulm \\
\hline
\end{tabular}

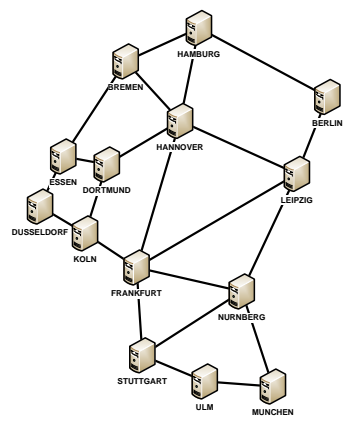

Fig. 3 DTAG reference network

Table 2 DTAG topology characteristics

\begin{tabular}{ll}
\hline Parameter & Value \\
\hline Number of Nodes & 14 \\
Number of links & 23 \\
Node degree & $3.29(\min .2$, Max. 6) \\
Link length $(\mathrm{km})$ & $186 \mathrm{~km}(\min .37$, Max:353 km) \\
Path length $(\mathrm{km})$ & $410 \mathrm{~km}(\min .: 37$, Max.:874) \\
Hop count & $2.35(\min : 1$, Max:5) \\
\hline
\end{tabular}

\subsection{Link capacity usage}

Figure 4 shows the total used link capacity required in the DTAG network for the different recovery mechanisms. If $w l_{l}^{w}$ is the number of working paths traversing link $l$ and $w l_{l}^{b}$ is the number of backup paths travers-

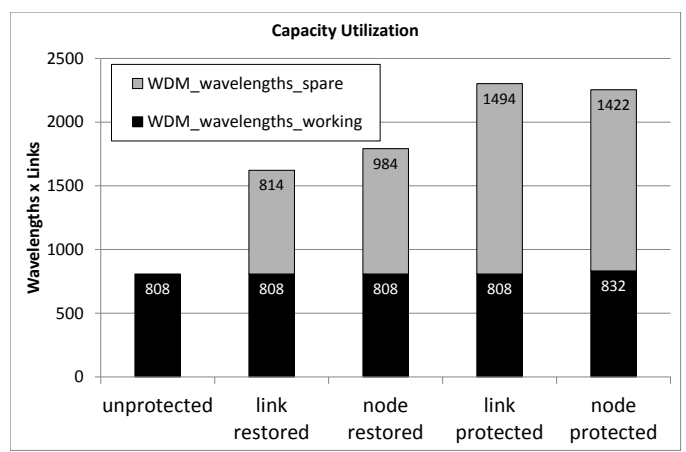

Fig. 4 Link capacity utilization for the DTAG topology

ing link $l$, the total used capacity (wavelengths $\mathrm{x}$ links, WL) for the network with $m$ links is calculated as

$\sum_{l=1}^{m} w l_{l}^{w}+w l_{l}^{b}$

These values are valid for both the transparent and opaque architectures, as the routing schemes used for both architectures are the same. Note that for $1+1$ protection the values are the same as for 1:1 protection and therefore not shown in the figure. Of course, in $1+1$ protection the spare wavelengths are always occupied; which is not the case for 1:1 protection, where the spare capacity can be used for low priority traffic.

We see that for the unprotected network we require 808 WL. If we want to be able to recover every lightpath in case of every possible link failure, we require an additional 814 WL (totaling $1622 \mathrm{WL}$ ) and for every possible link or node failure, 984 extra WL (totaling $1792 \mathrm{WL}$ ). Dedicated path protection clearly requires a lot more resources. In the DTAG topology, the capacity for the working paths in the link protection case is the same as the unprotected and restored cases, however, due to the absence of capacity sharing, the link protected-network requires 1494 WL extra and the node-protected network 1422 WL.

Figure 4 does not tell the complete story for the transparent network. In order to accommodate all the active lightpaths, the transparent network needs more wavelengths due to the wavelength continuity constraint [18]. If the wavelength channels on a link are numbered starting from 1 in increasing order (for instance, according to the ITU DWDM 50Ghz frequency grid [19]), and the highest used wavelength channel on link $l$ is $w c_{l}$, 
Table 3 DTAG topology : traffic matrix (Gb/s)

\begin{tabular}{|c|c|c|c|c|c|c|c|c|c|c|c|c|c|c|}
\hline Node ID & 1 & 2 & 3 & 4 & 5 & 6 & 7 & 8 & 9 & 10 & 11 & 12 & 13 & 14 \\
\hline 1 & 0.00 & 8.98 & 12.35 & 13.64 & 9.74 & 32.70 & 19.34 & 21.04 & 14.59 & 33.68 & 15.40 & 12.32 & 23.74 & 11.07 \\
\hline 2 & 8.98 & 0.00 & 5.76 & 6.23 & 4.51 & 14.19 & 9.92 & 10.56 & 6.59 & 12.59 & 6.43 & 5.13 & 10.15 & 4.69 \\
\hline 3 & 12.35 & 5.76 & 0.00 & 12.27 & 10.90 & 21.94 & 11.38 & 13.34 & 12.17 & 17.73 & 9.33 & 7.50 & 15.10 & 6.88 \\
\hline 4 & 13.64 & 6.23 & 12.27 & 0.00 & 12.52 & 24.58 & 12.48 & 14.31 & 18.02 & 19.54 & 10.42 & 8.33 & 16.96 & 7.68 \\
\hline 5 & 9.74 & 4.51 & 10.90 & 12.52 & 0.00 & 17.29 & 8.95 & 10.25 & 10.46 & 13.96 & 7.39 & 5.92 & 11.98 & 5.45 \\
\hline 6 & 32.70 & 14.19 & 21.94 & 24.58 & 17.29 & 0.00 & 28.99 & 33.09 & 27.13 & 47.75 & 26.20 & 21.64 & 27.56 & 19.88 \\
\hline 7 & 19.34 & 9.92 & $\begin{array}{l}11.38 \\
11.38\end{array}$ & $\begin{array}{l}12.48 \\
12.48\end{array}$ & 8.95 & 28.99 & 0.00 & 20.87 & 13.26 & 26.42 & 13.30 & 10.60 & 20.84 & $\begin{array}{l}9.65 \\
9.65\end{array}$ \\
\hline 8 & 21.04 & 10.56 & 13.34 & 14.31 & 10.25 & 33.09 & 20.87 & 0.00 & 15.16 & 30.04 & 14.81 & 11.94 & 23.42 & 10.79 \\
\hline 9 & 14.59 & 6.59 & 12.17 & 18.02 & 10.46 & 27.13 & 13.26 & 15.16 & 0.00 & 20.96 & 11.22 & 8.99 & 18.44 & 8.30 \\
\hline 10 & 33.68 & 12.59 & 17.73 & 19.54 & 13.96 & 47.75 & 26.42 & 30.04 & 20.96 & 0.00 & 22.38 & 18.38 & 34.50 & 16.09 \\
\hline 11 & 15.40 & 6.43 & 9.33 & 10.42 & 7.39 & 26.20 & 13.30 & 14.81 & 11.22 & 22.38 & 0.00 & 10.82 & 20.38 & 10.49 \\
\hline 12 & 12.32 & 5.13 & 7.50 & 8.33 & 5.92 & 21.64 & 10.60 & 11.94 & 8.99 & 18.38 & 10.82 & 0.00 & 16.32 & 7.82 \\
\hline 13 & 23.74 & 10.15 & 15.10 & 16.96 & 1198 & 27.56 & 2084 & 23.42 & 18.44 & 34.50 & 20.38 & 16.32 & 0.00 & 17.52 \\
\hline 14 & 11.07 & 4.69 & 6.88 & 7.68 & 5.45 & 19.88 & 9.65 & 10.79 & 8.30 & 16.09 & 10.49 & 7.82 & 17.52 & 0.00 \\
\hline
\end{tabular}

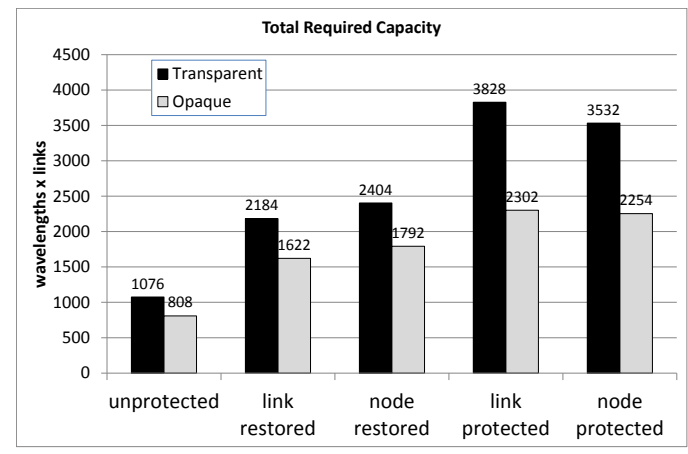

Fig. 5 Link capacity requirement for the DTAG Topology

then the total required capacity is calculated according to

$\sum_{l=1}^{m} w c_{l}$

For the opaque solution this amounts to the sum of the working capacity and spare capacity from Figure 4, all wavelengths which are available are also used. We immediately see that, when compared to the opaque solution, the transparent solution requires a lot more resources. For the unprotected case, we require 1076 WL (or 33\% more resources), meaning that the links are utilized only for $75 \%$ (the active lightpaths consume $808 \mathrm{WL}$ ) due to the wavelength continuity constraint. For the node restored case the situation is similar $(35 \%$ extra resources or $74 \%$ link utilization). For the link protected case, the increase is much more prominent, requiring almost $66 \%$ extra resources due to the wavelength continuity constraint (60\% link utilization). This is due to the longer paths required for the backup paths, since they have to be disjoint from the working paths. If we compare the solutions for node protection to node restoration, we see a $21 \%$ (1792 WL vs. $2254 \mathrm{WL}$ ) decrease in link resource consumption for the opaque case and an even larger $32 \%$ reduction in WL consumption ( $2404 \mathrm{WL}$ vs. $3532 \mathrm{WL}$ ) in the transparent case. We can attribute this to the wavelength continuity constraint in transparent networks.
There is one peculiarity to these results. The attentive reader will undoubtedly have noticed that, in contradiction to common sense, the link-protected solution consumes more resources than the node-protected solution. This is due to the four nodes in close vicinity of each other in the DTAG topology (the link-length distribution is not smooth) and the fact that we use a physical length shortest cycle, which in some cases routes through these nodes. If we use hop count instead of physical length in the routing algorithm, this does not occur.

\subsection{Node capital expenditures}

Now that we have shown a significant reduction in wavelength consumption for restoration compared to protection for both the opaque and transparent network architectures, we turn our attention to the Capital Expenditures (CapEx) of the nodes. The CapEx model of the nodes is given in Table 1 and broken down in three main components:

- Transmission. These are the source and destination transponders (transparent) / transceivers (opaque), any transponders/transceivers in intermediate nodes and the OTN linecards (opaque).

- Switching. These are the switching fabric and AWG/terminals in the ROADM (transparent) or the backplane (basic node) in the OTN cross-connect (opaque).

- Tributaries. The transmission equipment (transponders or transceivers) towards the client host or network.

Figures 6 and 7 show the CapEx results for the transparent and opaque solutions respectively. It is immediately clear that the restoration (i.e. the baseline solution for shared mesh protection) is cheaper than $1: 1$ or $1+1$ protection in both solutions. In the transparent network, the cost difference is in the switching. If the capacity increases (between different recovery methods), the capacity of some links may exceed the number of wavelengths (80), so some nodes need a parallel line 
system (an extra ROADM degree) in order to accomodate this increase in traffic. For the opaque solution, the main cost is in the transmission equipment because we need 2 transceivers in every intermediate node for each traversing connection. There is also an increase in the switching cost due to larger backplane (basic node) requirements for protection when compared to restoration. Also, in the opaque solution, we see that the cost of the tributaries is negligible compared to the overall node cost.

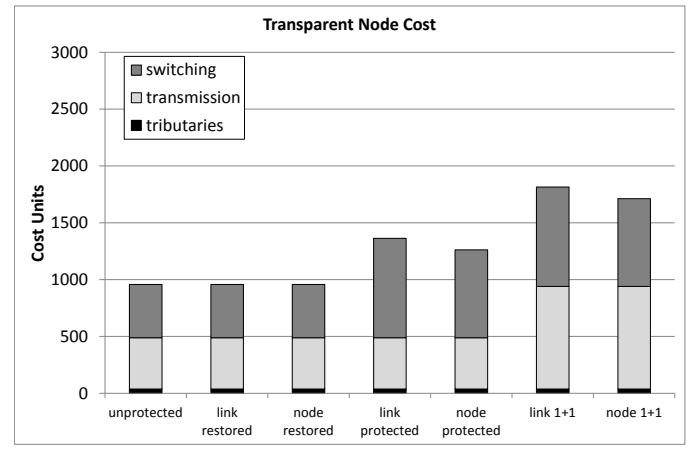

Fig. 6 Transparent node cost

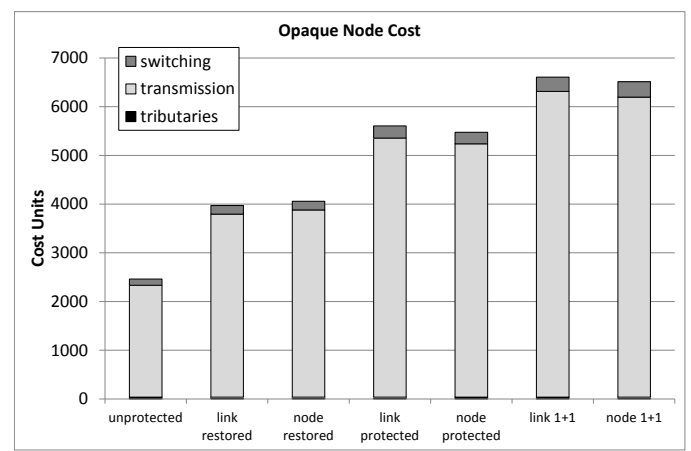

Fig. 7 Opaque node cost

If we compare the gain by implementing protection sharing/restoration, we see that for the opaque solution, the CapEx reduction is roughly 26\% (4059.3 Cost Units (CU) vs $5474.9 \mathrm{CU}$ ), while for the transparent solution it is roughly $24 \%$ (958 CU vs $1261.6 \mathrm{CU}$ ). This result contradicts our previous results from [11], where we found no such advantage for the traffic from Table 3 and only a little advantage if we doubled the amount of traffic in the traffic matrix. The reason for the discrepancy lies in the fact that we did not take into ac-

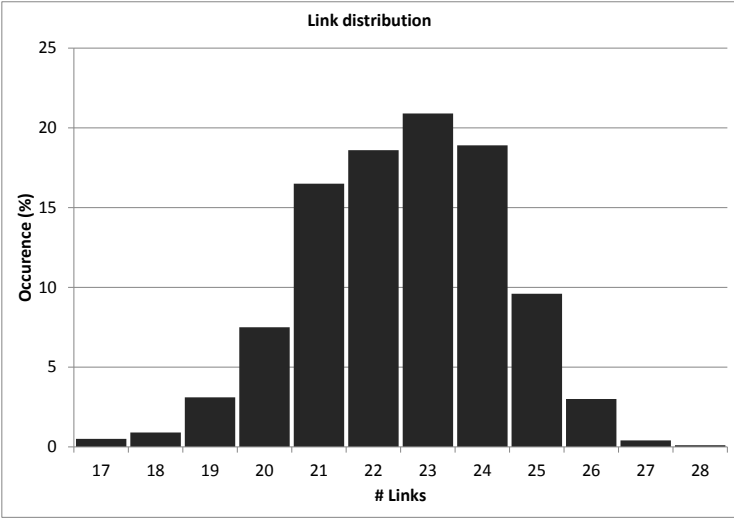

Fig. 8 Link distribution for the generated topologies

count the wavelength continuity constraint in our first work. As was shown in Figure 4 the continuity constraint leads to almost $33 \%$ increase in traffic for the restoration case and a $66 \%$ increase in traffic for the protection case, effectively increasing the reduction in node CapEx gained by resource sharing in transparent networks. While we expected a small increase from including the wavelength continuity constraint, we never expected such a significant one. This find lead us to perform more extensive research in order to find the relation between the traffic and the node cost for transparent and opaque networks. This shows that a reduction in wavelength consumption is definitely not a direct indicator for a similar reduction in network cost. The CapEx reductions are far less outspoken than the wavelength consumption, moreover, where the wavelength consumption decrease was largest in the transparent network, the node cost decrease is larger in the opaque network. In the next section, we perform a thorough investigation how the node CapEx gain (through the introduction of resource sharing) scales with traffic demand and network meshedness in a randomized scenario.

\section{Randomized study}

In order to have a more meaningful analysis and evaluate the benefits of resource sharing more thoroughly, we extend our dimensioning study by using random generated 14-node networks as opposed to the single reference network from the previous section. We number the nodes 1-14 at random, and apply the traffic matrix from Table 3 to each of these networks to calculate and analyze the node CapEx. 


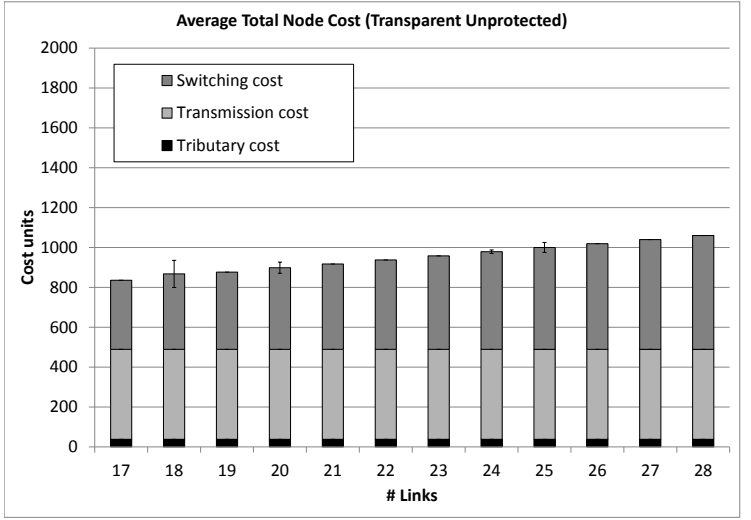

Fig. 9 Average node cost, transparent unprotected

We generated 2-node-connected planar graphs by randomly assigning 14 points to an $800 \mathrm{~km}$ by $800 \mathrm{~km}$ grid and computing the Gabriel graph [20] for these 14 points. We discarded all non 2-connected graphs until we had a population of 1000 random graphs. These graphs had a link distribution shown in Figure 8. It seems that the topology with 23 links (like the DTAG topology) is the most likely to occur.

\subsection{Influence of the Topology}

In this section we investigate the influence of the number of links and the topology on the node cost in both transparent and opaque networks. The results show that, for the traffic matrix from Table 3, for the transparent solution, the node CapEx increases for unprotected traffic, but is more or less stable if we apply resiliency. In the opaque solution, the node CapEx always goes down with increasing node degree.

Figure 9 shows the average node costs for the generated networks versus the number of links in the generated topologies for unprotected routing. $2 \sigma$ confidence intervals are included (note that there is no variation for most of the unprotected networks). The cost for tributaries (451.2 CU) and the transponders (451.2) is the same for all solutions. Indeed, all networks (17-28 links) are transparent for all shortest paths. The cost of a transparent network goes up (from $836 \mathrm{CU}$ to 1060 $\mathrm{CU})$ with the number of available links. From Figure 9 we clearly see that this is due to an increase in switching cost (from $347 \mathrm{CU}$ to $571 \mathrm{CU}$, a $63 \%$ increase), or more specifically, an increase in the degree of the ROADM node due to the increase in physical degree of the topology. For some networks (the 18, 20, 24 and 25) there is a slight variation in the cost of the switch due to some network topologies requiring parallel line

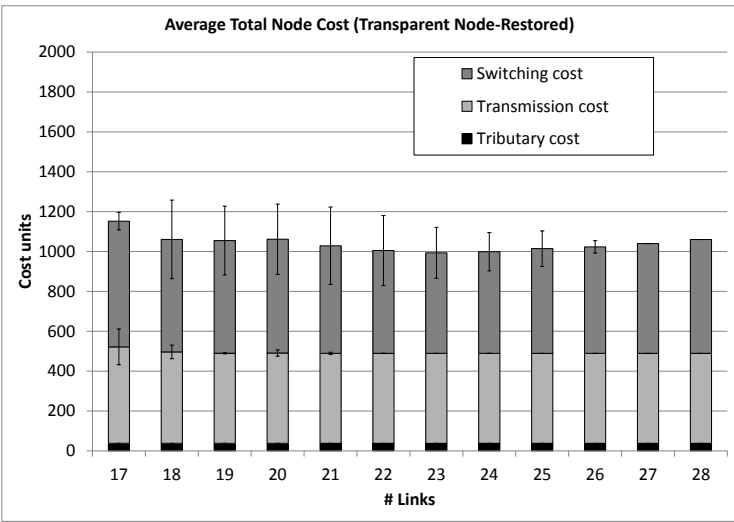

Fig. 10 Average node cost, transparent node-Restored

systems. However, we can conclude that for this level of traffic, there is very little influence of the actual topology and only the number of links (and thus the average node degree) affects the node CapEx of the network.

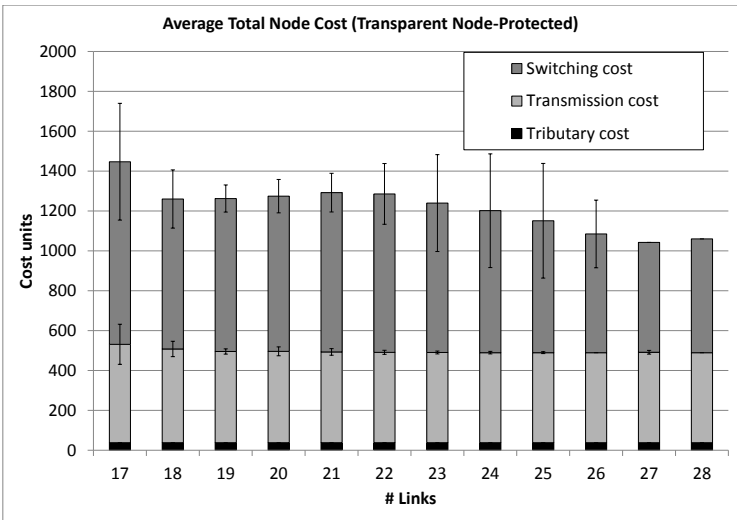

Fig. 11 Average node cost, transparent node-protected

Figures 10 and 11 show similar figures for node restoration and protection. We see that the monotonous increase of the cost vs. the number of links observed for the unprotected case is not present anymore and the node CapEx shows a more flat distribution with respect to the number of network links (the average node degree). In Figure 10, the transmission cost slightly decreases (484 CU to $451 \mathrm{CU}$ ) with an increase in links. This is somewhat expected, as the restoration path will be longer in sparse networks, requiring regeneration, which is implemented by terminating and continuing the traffic at an intermediate node, which means the need for additional transponders. The variation in the switching cost is also more present than in the unprotected case, meaning there is more dependence on the actual topology. We will investigate the effect of traffic 


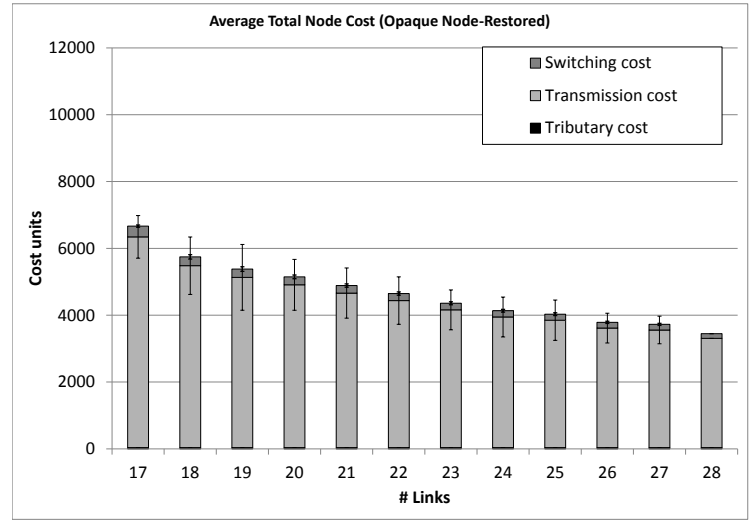

Fig. 12 Average node cost, opaque node-restored

increases in the next section.

Figure 11 shows the same behavior for the transmission cost. It goes slightly down with an increase in the number of links (from $493 \mathrm{CU}$ to $451 \mathrm{CU}$ ). The transmission cost is slightly higher compared to the restored / unprotected case due to an additional increase in the length of the working/backup paths due to the use of a shortest cycle algorithm (vs. shortest path on the remaining topology after a failure in the restoration scenario).

If we look at the cost benefits of restoration vs protection (i.e. the difference between Figure 11 and Figure 10) we see that, for the 23 link network, in our generated topologies the gain is around 25\% (993 CU vs 1240 $\mathrm{CU})$. What is very peculiar is that the gain is higher for the medium meshed networks (21-24 link networks are all in the $20-25 \%$ range) than for the higher meshed networks (the gain for the 26 links network is already less than $10 \%$ ).

We now turn our attention to the opaque architecture. Figures 12 and 13 show the node costs for the node-restored and node-protected cases. We see that the cost of the network scales down with an increase in the number of links. This is because an increase in meshedness reduces the average hops on each path, which in turn reduces the number of $\mathrm{O} / \mathrm{E} / \mathrm{O}$ conversions and therefore the transmission cost. The cost reduction is almost $50 \%$, with a node cost of $6666 \mathrm{CU}$ for the 17 link network and a node cost of $3448 \mathrm{CU}$ for the 28 link network. There is again little variation due to the actual topology as the $2 \sigma$ confidence intervals are quite small, the larger values for 17, 18 and 27 (and infinite for 28) are due to the small data set for these networks.

When we compare the two solutions (restoration vs. protection), we again see a significant node CapEx gain

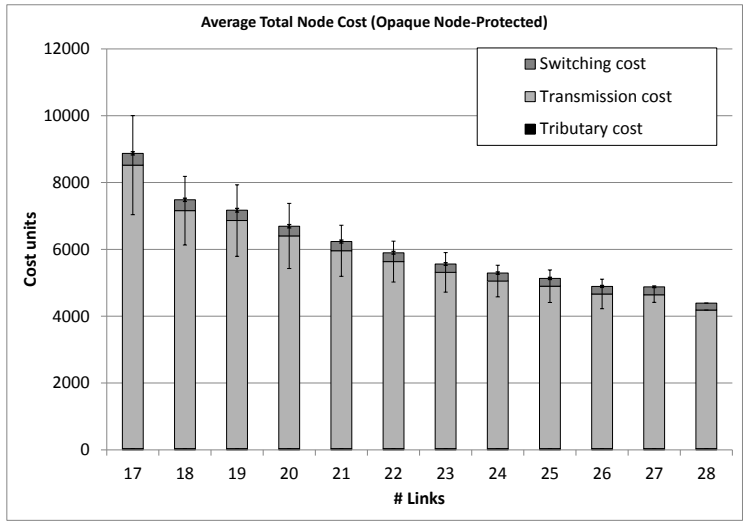

Fig. 13 Average node cost, opaque node-protected

due to protection sharing which decreases slightly with the number of links in the network. The gain is $25 \%$ for a 19 link network (5380 CU vs $7171 \mathrm{CU}$ ) and $23 \%$ for a 26 link network (3785 CU vs. $4890 \mathrm{CU}$ ).

\subsection{Influence of Traffic Scaling}

In order to evaluate the effects of the traffic load, we scaled the traffic from Table 3 from $50 \%$ to $500 \%$ in $50 \%$ increments. From a multiplier of $3-3.5 x$ onwards, the ROADM degree of some node exceeds 19 (which is the limit set by the use of $1 \times 20 \mathrm{WSSs}$ ), and the OTN backplane reaches its limits (128 slots) at $4.5-5 \mathrm{x}$. We therefore limit our results to a traffic multiplier for $3 \mathrm{x}$ for the transparent case and $4.5 \mathrm{x}$ for the opaque case.

From Figure 14 it is clear that the increase in node cost with the number of links for transparent networks we noticed in the previous subsection is only valid for the low traffic cases where there is little increase in ROADM degrees through the necessity for additional parallel line systems. The slightly increasing slope for multiplier values $0.5 \mathrm{x}$ and $1 \mathrm{x}$ turns to a fairly constant line for a multiplier of $2 \mathrm{x}$ and becomes decreasing if we further increase the multiplier. The figure also shows that the dependency on the topology is independent of an increase in traffic. The $2 \sigma$ confidence intervals on the cost become larger the further we scale the traffic, but the increase is linear, always around $14 \%$ of the traffic value. We only show the confidence intervals for $0.5 \mathrm{x}$, $1 \mathrm{x}, 2 \mathrm{x}, 3 \mathrm{x}$ and $4 \mathrm{x}$ in order to avoid cluttering the figures. The only exception to this $14 \%$ rule is the bottom line in Figure 14, where there is no variation. Due to the low amount of traffic, all traffic could always be routed transparently (except for the 17 and 18 node networks 


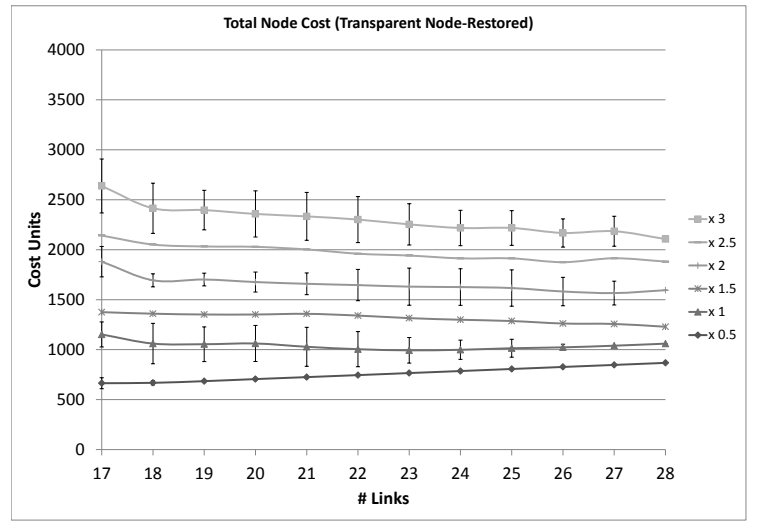

Fig. 14 Total node cost for different traffic multipliers, transparent node-restored

which show very small variation). Figure 15 shows the same data, but for the protection case.

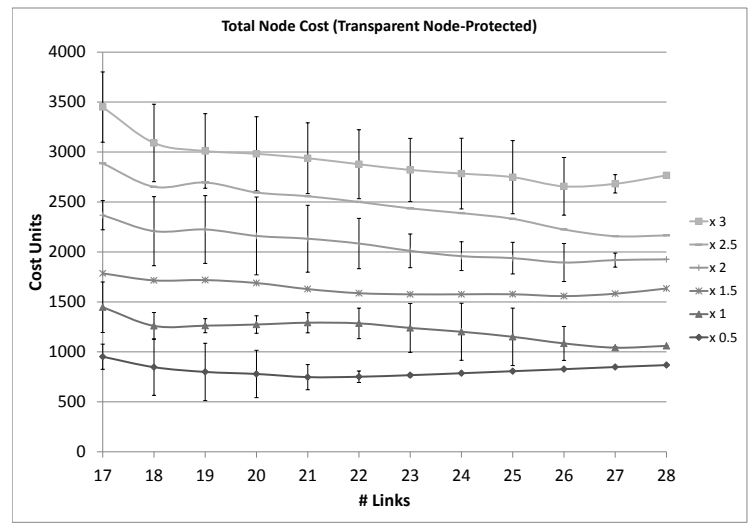

Fig. 15 Total node cost for different traffic multipliers, transparent node-protected

We summarize the relative CapEx gain for the transparent networks in Fig. 16. We include the networks with 20-25 links and apply the traffic multiplier from $0.5 \mathrm{x}$ to $3 \mathrm{x}$. We omit the other cases because of some ROADM degrees exceeding the limits set by the use of $1 \times 20$ WSSs as noticed before. What we learn from this figure is that the relative gain through protection sharing in transparent networks is very dependent on the traffic scaling. For low traffic there is almost no resource gain (less than $5 \%$ for the 23 node network). From the moment the traffic loads exceeds a certain threshold (here it's roughly at the $1 \mathrm{x}$ multiplier), the average ROADM degree in the network goes up and the relative decrease in traffic load needed to reduce the degree goes down. In turn, the probability of this happening goes up significantly. If we have a node with 3 neighbours in the physical topology, reducing it from

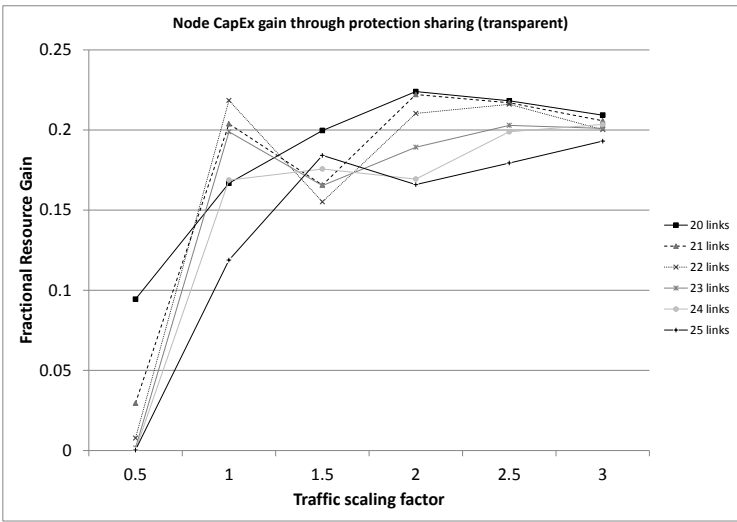

Fig. 16 CapEx gain through resource sharing in transparent networks

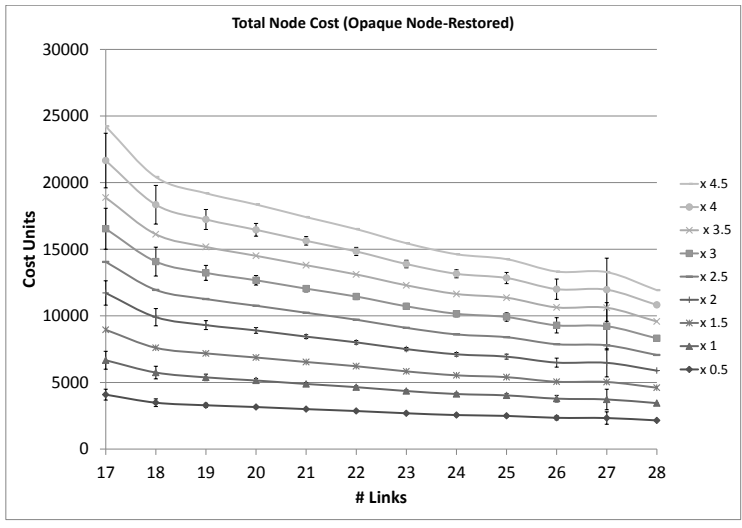

Fig. 17 Total node cost for different traffic multipliers, opaque node-restored

a 14-degree to a 12-degree ROADM takes less of a relative traffic reduction than to reduce it from a 4-degree to a 3-degree ROADM. The wavelength continuity constraint is certainly an important contributor in speeding up this process. After this threshold is reached the CapEx gain of resource sharing is roughly $17-22 \%$.

For opaque networks, the overall picture is quite different. As shown in Figs. 17 and 18, the total node cost always goes down with the number of links in the network, no matter the load. Also, it is independent of the load. For instance, in the node-restored case the relative gain from 20 links to 25 links is $21 \%$ for $0.5 x$ traffic $(2490 \mathrm{CU}$ vs $3156 \mathrm{CU})$ and also $21 \%$ (12839 CU vs $16457 \mathrm{CU}$ ) for $4 \mathrm{x}$ traffic. It's also independent of the actual underlying topology, since the confidence intervals are very small (less than $3 \%$ overall). This decreasing trend has as a result that operators will be able to find an optimum between the additional link cost (for increasing the node degree) and the decreasing node cost (due to the shorter paths in the network). Remember 


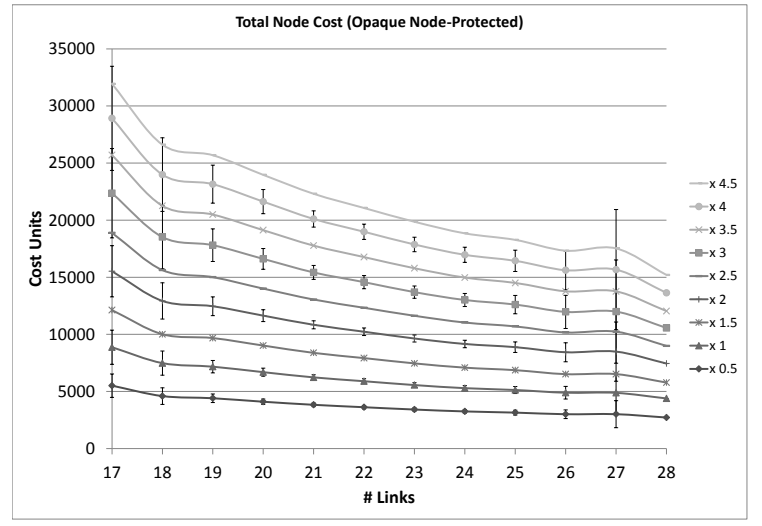

Fig. 18 Total node cost for different traffic multipliers, opaque node-protected

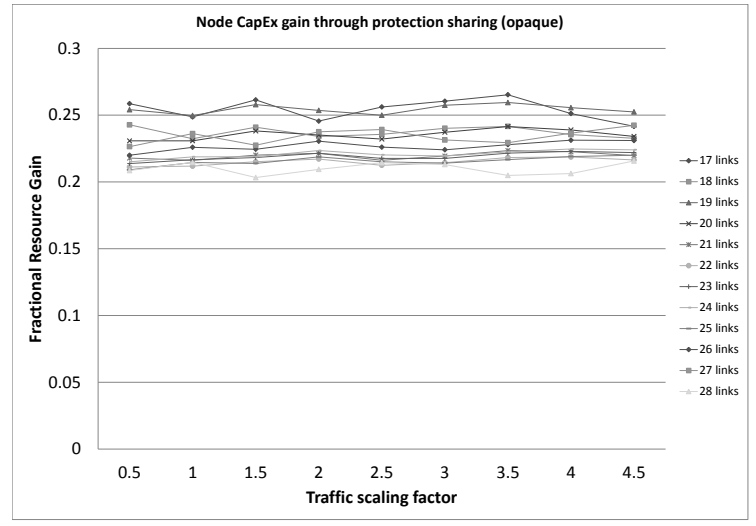

Fig. 19 CapEx gain through Resource Sharing in opaque Networks

that transparent networks with low traffic do not have this and have a decrease in node cost together with a decrease in link cost, always driving the optimum towards sparsely meshed networks. This may give transparent network operators additional incentives to prefer higher bandwidths per channel and more wavelengths per fiber instead of installing parallel line systems.

When comparing the overall cost reduction from Figure 16 and 19 we see that the node CapEx reduction for transparent networks in the high load case (17-22\%) is definitely comparable to the CapEx reduction in the opaque architecture (21-25\%). We find this quite surprising as the load reduction through resource sharing in transparent networks only affects the ROADM degree (See Figure 10) and therefore only the cost of the WSS drives this reduction. In opaque networks the cost reduction is driven through a reduction in the number of required transceivers, linecards and a reduction in the size of the switching fabric.

\section{Conclusions and future work}

In this paper, we evaluated the impact of transparent node architectures on the benefits that shared path protection brings with regard to network CapEx. We performed a thorough investigation into the possible CapEx saving through resource sharing in nation-wide transparent and opaque transport networks. The number of links in the network has a small impact on this gain, with sparsely meshed networks having greater benefit than densely meshed networks. We find that the load has an important impact in transparent networks, where low load (i.e., few parallel line systems) means that the network does not benefit greatly from protection sharing. However, when the average required ROADM degree increases, the CapEx benefits approach the same levels as for traditional opaque networks. Opaque networks do not show a dependency on the load and always have a similar node CapEx gain from protection sharing. We can thus conclude that traditional opaque networks will have a CapEx reduction in line with capacity requirement reductions due to resource sharing. However, for transparent networks, this relationship is not true, especially for low loads. With the ongoing trend towards higher bit rates $(400 \mathrm{~Gb} / \mathrm{s}$ and up), denser channel spacing and more efficient spectrum usage (Flexigrid), we think the balance for transparent networks will tip over towards this low load solution, meaning protection sharing may be less interesting to implement in the optical layer of such networks.

Acknowledgements This work was supported by the European Commission within the STRONGEST-project (Scalable, Tunable and Resilient Optical Networks Guaranteeing Extremely-high Speed Transport), funded through the 7th ICT-Framework Programme.

\section{References}

1. B. Ramamurthy et al., Transparent vs. opaque vs. translucent wavelength-routed optical networks, Proc. OFC'99 (1999).

2. A. Morea et al., A critical analysis of the possible cost savings of translucent networks, Proceedings Design of Reliable Communication Networks DRCN (2005).

3. R. D. Doverspike et al., Future Transport Network Architectures, IEEE Communications Magazine,Special Issue on Reliable Communication Networks, (1999).

4. A. Jarray et al., CAPEX/OPEX effective optical wide area network design, Telecommunications Systems, 49(3), 329$344(2012)$.

5. A. Ferreira et al., Models, Complexity and Algorithms for the Design of Multi-fiber WDM Networks, Telecommunications Systems, 24, pp. 123-138 (2003). 
6. R. Ramaswami and K.N. Sivarajan,Routing and Wavelength Assignment in All-Optical Networks, IEEE/ACM Transactions on Networking, 3(5), 489-500, (1995).

7. Sungwoo Tak and E.K. Park, Restoration performance study of k-shortest disjoint paths in WDM optical networks, Telecommunications Systems, 34, pp. 13-26 (2007).

8. J. W. Suurballe and R. E. Tarjan,A quick method for finding shortest pairs of disjoint paths, Networks, 14, 325336, (1984).

9. P.-H. Ho et al., Spare capacity allocation for WDM mesh networks with partial wavelength conversion capacity, Proceedings High Performance Switching and Routing, HPSR, (2003).

10. Y. Liu et al., Approximating Optimal Spare Capacity Allocation by Successive Survivable Routing,IEEE/ACM Transactions on Networking, 13(1), 198-211, (2005).

11. D. Staessens et al. Cost Efficiency of Protection in Future Transparent Networks, Proceedings of International Conference on Transparent Optical Networks ICTON, (2009).

12. Y. Ishii et al., MEMS-based 143 wavelength-selective switch with flat passband, 35th European Conference on Optical Communication, ECOC (2009)

13. M. Gunkel et al., A Cost Model for the WDM Layer, Proceedings of Photonics in Switching (2006).

14. R. Huelsermann et al., Cost modeling and evaluation of capital expenditures in optical multilayer networks, OSA Journal of Optical Networking, 7(9), (2008).

15. M. De Groote et al., Cost comparison of different Translucent Optical Network Architectures, Proceedings Conference of Telecommunication, Media and Internet TechnoEconomics CTTE (2010).

16. STRONGEST: Scalable, Tunable and Resilient Optical Networks Guaranteeing Extremely-high Speed Transport, http://www.ict-strongest.eu/.

17. M. Pickavet et al., Recovery in multilayer optical networks, Journal of Lightwave Technology, 24(1), 122-134, (2006).

18. C. Fenger, Performance evaluations for Dynamic Wavelength Routed All-Optical Networks Telecommunication Systems, 25, 117-127 (2004).

19. ITU-T recommendation 694.1 Spectral grids for WDM applications: DWDM frequency grid, http://www.itu.int.

20. K. R. Gabriel and R. R. Sokal, A new statistical approach to geographic variation analysis, Systematic Zoology (Society of Systematic Biologists) 18 (3) pp. 259270, 1969 